\title{
Medicina basada en la evidencia (MBE). Ventajas
}

\author{
LM. Junquera ${ }^{1}$, J. Baladrón², J.M. Albertos ${ }^{3}$, S. Olay ${ }^{4}$
}

\begin{abstract}
Resumen: La Medicina basada en la Evidencia (MBE) se define como un proceso cuyo objetivo es la selección de los mejores argumentos científicos para la resolución de los problemas que la práctica médica cotidiana plantea. Filosóficamente es un nuevo paradigma (verificacionista), que se ha extendido a todas las profesiones sanitarias (Cirugía Basada en la Evidencia, Odontología Basada en la Evidencia, etc.), soportado por los avances tecnológicos. Aunque sus ventajas son evidentes, también se han reconocido limitaciones, especialmente en lo concerniente a la práctica quirúrgica. En el presente trabajo se revisan los fundamentos y principales ventajas de la MBE.
\end{abstract}

Palabras clave: Medicina basada en la evidencia; Cirugía basada en la Evidencia; Revisiones sistemáticas; Ventajas.

\begin{abstract}
Evidence Based Medicine (EBM) is defined as a process whose objective is the selection of the best scientific arguments for the resolution of problems in the medical practice. Phylosophically, it is a new paradigm that has been extended to all the sanitary professions (Evidence Based Surgery, Evidence Based Dentistry, etc.), sustained by the technological advances. Although their advantages are evident, also there have been recognized limitations, specially concerning the surgical practice. At the present work the bases and the main advantages of the EBM are checked.
\end{abstract}

Key words: Evidence Based Medicine; Evidence Based Surgery; Systematic reviews; Advantages.

\section{Introducción}

La Medicina basada en la Evidencia (MBE), término acuñado por Gordon Guyatt, ${ }^{1}$ se define como un

\footnotetext{
1 Profesor Titular Vinculado de Cirugía Oral y Maxilofacial. Universidad de Oviedo. Hospital Universitario Central de Asturias, España.

2 Cirujano Oral y Maxilofacial. Practica Privada, Oviedo, España

3 Médico Adjunto. Servicio de Cirugía Oral y Maxilofacial. Hospital Xeral. Santiago de Compostela, España.

4 Profesora Asociada. Universidad de Oviedo. Escuela de Estomatología, Asturias. España.

\section{Correspondencia:}

L.M. Junquera

Universidad de Oviedo. Escuela de Estomatología

Catedrático José Serrano s/n

33009 Oviedo, España.

E-mail: Junquera@sci.cpd.uniovi.es
}

proceso cuyo objetivo es el de obtener y aplicar la mejor evidencia científica en el ejercicio de la práctica médica cotidiana. Para ello, se requiere la utilización concienzuda, juiciosa y explícita de las mejores 〈evidencias»disponibles en la toma de decisiones sobre el cuidado sanitario de los pacientes. ${ }^{2}$ En nuestro idio$\mathrm{ma}$, se entiende por evidencia la certeza manifiesta sobre una cosa que elimina cualquier duda racional sobre la misma. Algunos autores, prefieren la utilización del término Medicina Basada en Pruebas, considerando a estas últimas como los argumentos o razones que demuestran una cosa. Desde esta perspectiva el proceso consistiría en la selección de los mejores argumentos científicos para la resolución de los problemas que la práctica médica cotidiana plantea.

Actualmente, se tiende hablar más de Atención sanitaria basada en la Evidencia, que englobaría la Medicina basada en la Evidencia, Enfermería basada en la 
Evidencia, Odontología basada en la Evidencia y así todas las distintas especialidades y/o profesiones que intervienen en la atención sanitaria de los pacientes.

\section{Antecedentes históricos. Fundamentos. Ventajas}

Históricamente la Medicina basada en la Evidencia tiene un doble origen: filosófico y tecnológico.

El origen filosófico de la esta nueva tendencia se remonta a mediados del siglo XIX y tiene en Paris, con sus escépticos post-revolucionarios (Bichat, Magendie, etc. $)^{3}$ su punto de partida. El nuevo paradigma que esta nueva corriente impone se sustenta en una concepción de la medicina teórica basada en la experimentación y una medicina práctica basada en la verificación o validación. Hasta llegar a este nuevo paradigma la ciencia médica tuvo que recorrer un largo camino. Desde la perspectiva Aristotélica y su ulterior influjo, que discernía entre saber especulativo (universal, cierto) y saber práctico (particular, probable), hasta la concepción imperante a partir del siglo XVII de una medicina teórica basada en la experimentación y una medicina práctica sustentada por la extrapolación.

Para la medicina Aristotélica, el conocimiento en la práctica médica no puede ser nunca cierto sino sólo probable, entendiendo como probabilidad aquella opinión (doxa) que defienden las gentes más sabias y expertas (probabilidad subjetiva de hoy en día). La toma de decisiones en esta línea de pensamiento está condicionada por la autoridad de quienes defienden una postura y, por tanto, no con la evidencia sino con la intención. ${ }^{4}$ Con la llegada del siglo XVII Bernoulli elabora la teoría matemática de las probabilidades (probabilidad objetiva), que sustituye la idea de certeza por la de probabilidad. Con este nuevo teorema, se hizo frente a dos cuestiones preocupantes desde la Edad Media: 1. ¿Cuál era la postura a tomar, cuando dos opiniones opuestas estuvieran defendidas exactamente por el mismo número de personas sabias (equiprobabilismo)?; 2 ¿Podría ser admitida como válida sobre un tema concreto, la opinión de un solo autor sabio y experimentado? Frente a la primera cuestión se admitía, que ambas opiniones, a pesar de ser opuestas, podrían ser válidas. Para el segundo interrogante, también se consideraba que la opinión de un único sabio, debería ser considerada como prudente. La teoría de la probabilidad objetiva permitió calibrar el nivel de certeza, asignándole un valor objetivo (50\%, $1 \%$, etc.) de posibilidades de veracidad. A partir de este momento, la ciencia empírica o experimental dejó de ser un saber deductivo, para convertirse en inductivo, naciendo lo que Claude Bernard llamó medicina experimental. ${ }^{4}$

El gran inconveniente de esta línea de pensamien-
Tabla 1. Principales ventajas de la MBE para el profesional de la salud

- Disminución de la amplia variabilidad (injustificada) en la atención médica.

- Reducción de la brecha entre la generación del conocimiento y su aplicación.

- Superación de modas, propagandas, inducciones y otras formas de imposición.

- Estimulación de la evaluación crítica del conocimiento establecido.

- Estímulación para la práctica reflexiva.

- Facilitación del aprendizaje de las estrategias de búsqueda y recuperación de la información.

- Promoción de la capacidad de discernir entre información científica y no científica.

- Promoción del establecimiento de un sistema propio de educación continuada.

- Promoción de la interconexión entre la atención médica, la educación y la investigación.

- Favorece la apreciación del valor de la verdad.

- Favorece la apreciación del valor de la mejor alternativa.

- Eliminación de las alternativas que no representan las mejores opciones para los pacientes de acuerdo con el avance científico y tecnológico.

to deriva del hecho de que el saber práctico se adquinía exclusivamente por extrapolación del conocimiento teórico o experimental. Un error teórico (la barrera placentaria es impermeable a los medicamentos), conllevó por extrapolación los perniciosos efectos de la talidomida en miles de recién nacidos. ${ }^{4}$

Con la llegada del siglo XX la medicina experimental se hizo más humilde, comenzando a ganar peso, lo que se denominó investigación clínica, sustituyéndose los conceptos de analogía y extrapolación por los de verificación y evidencia. Los ensayos clínicos, la epidemiología clínica y el desarrollo de Internet fueron las herramientas que fomentarían, el cuerpo doctrinal de lo que hoy conocemos como MBE. ${ }^{5}$

Desde un punto de vista tecnológico, un poderoso germen del ulterior desarrollo de la MBE fue la Escuela de Medicina de la Universidad McMaster en Hamilton, Ontario, Canadá. A finales de los años sesenta se comenzó a desarrollar en esta Universidad, un programa educativo interdisciplinario centrado en la resolución de problemas individuales. ${ }^{6}$ Este tipo de aprendizaje desarrolla, como después veremos, una metodología muy similar a la propia de la MBE: identificación del problema, búsqueda de información sobre el mismo, y resolución del problema en consonancia con la información recogida. Figura clave en el desarrollo de esta Escuela de Medicina, y uno de los pioneros en la divulgación y práctica de la MBE, fue el estadounidense David Sackett, quien enfatizó sobre la importancia de la epidemiología y el conocimiento estadístico, antes de aplicar sus teorías en el ejercicio de la práctica médica, siguiendo la comiente de la MBE. Otra figura trascendente de este nuevo 
paradigma fue el epidemiólogo británico Archie Cochrane, quien preocupado por la falta de trabajos controlados sobre la práctica médica, impulsó a un grupo de investigadores de la Universidad de Oxford, entre otros a Iain Chalmers, hacia la elaboración de una base de datos que recogiera revisiones sistemáticas de trabajos controlados y randomizados, de donde se originaría lo que actualmente se conoce como Colaboración Cochrane (con centros diseminados por todo el mundo $)^{7-9}$ y también The Cochrane Library, obra de edición periódica en soporte electrónico de amplia difusión por Internet, en la que se recoge el trabajo de diferentes grupos de revisores e interesantes metaanálisis. Las ventajas de la MBE deben contemplarse desde una triple perspectiva: ventajas para el profesional de la salud (Tabla 1), ventajas para el paciente, al ofrecerle la mejor altemativa (diagnóstica, terapéutica) existente, y beneficios para el sistema sanitario en su conjunto, al identificar y suprimir de forma mas efectiva los cuidados ineficaces.

\section{Metodología de la MBE}

Frente al ejercicio médico cimentado por la autoridad de expertos, más o menos próximos, y las observaciones clínicas no sistematizadas (experiencia personal), el nuevo paradigma de la MBE mantiene un alto valor a la experiencia clínica, pero la vincula indisolublemente con la mejor evidencia científica publicada sobre el problema concreto del paciente. En opinión de Sackett y cols., ${ }^{2}$ practicar la MBE significa integrar la competencia clínica individual con la mejor evidencia clínica externa disponible a partir de la investigación sistemática. Por competencia o maestría clínica individual se quiere significar la habilidad y el buen juicio que el clínico adquiere a través de la experiencia y la práctica cotidiana. La mayor competencia se puede reflejar de muchas maneras, pero sobre todo en la realización de diagnósticos más efectivos y eficientes (evitando la multiplicación de estudios innecesarios, que vemos tan habitualmente), y en un trato humano que contemple los problemas, derechos y preferencias del paciente a la hora de tomar decisiones clínicas sobre su cuidado. La metodología a utilizar para el desarrollo de la MBE debe seguir al menos cuatro pasos: ${ }^{10-15}$

\section{Formular de manera precisa una pregunta a partir del problema clínico del paciente}

Consiste en convertir los interrogantes que surgen durante la historia clínica y la exploración, y que percibimos como necesidades de información, en una pregunta, simple y claramente definida. Lógicamente estos interrogantes pueden afectar al diagnóstico, técnicas complementarias del mismo, tratamiento, pro-
Tabla 2. Principales fuentes para el ejercicio de la MBE

- ACP Journal - American College of Physicians (www.acponline.org)

- Evidence Based Medicine - British Medical Journal (www.bmj.com)

- Evidencia en Atención Primaria - Fundación MF (www.fundacionmf.org.ar)

- Evidence Based Health Care Managment - Churchill Livingstone- Clinical Evidence- BMJ publishing group (www.bmj.com)

- Best evidence - BMJ Publishing group (www.evidence.com)

- Bandolier (www.jr2.ox.ac.uk/bandolier/)

- York University (www.york.ac.uk/inst/crd/)

- POEMS

(www.medicalinforetriever.com/poems/poemsearch.cfm)

- Trip Database (http://www.tripdatabase.com/index.cfm)

nóstico, etc. Por ejemplo: un varón de 70 años, presenta una tumoración en cola de parótida de 5 años de evolución y comportamiento asintomático. La experiencia clínica tras la exploración, puede orientar un diagnóstico de presunción: tumor benigno de glándula parotídea. ¿Qué estudios complementarios me pueden confirmar con mayor fiabilidad este diagnóstico?

\section{Localizar las pruebas disponibles en la literatura, siguiendo una estrategia}

La búsqueda de la literatura relevante a la pregunta se realiza en bases de datos bibliográficas, de las cuales la más utilizada y conocida es MEDLINE. Hoy en día se puede acceder fácilmente a esta base de datos a través de Internet (Pubmed). Con la ayuda de estrategias de búsqueda apropiadas se puede obtener información (parcial o completa) de manera rápida y sencilla. Sin embargo, la estrategia de búsqueda empleada (a veces se precisa de más de una), y la selección de la información sobre el interrogante concreto que nos ocupa, puede estar sujeta a error si el profesional de la salud no dispone de un sentido crítico sobre los contenidos publicados.

Otras fuentes como las revistas secundarias o de resúmenes como el ACP Journal Club, Evidence Based Medicine y Evidence-Based Practice que seleccionan y resumen, con los criterios de la MBE, lo mejor de lo publicado, pueden ayudar a minimizar este problema (Tabla 2). En este sentido, merece especial mención la denominada Colaboración Cochrane que tiene por objetivo preparar, mantener y difundir revisiones sistemáticas sobre los efectos de la atención sanitaria», ${ }^{16}$ y que para ello publica una base de datos de revisiones sistemáticas sobre muchos aspectos de la práctica médica. Actualmente, más de 5.000 personas de más de 50 países están colaborando con esta empresa (Figs. 1 y 2). De su importancia da una idea el hecho de que las principales revistas médicas estén dispuestas a publicar versiones adaptadas de revisio- 


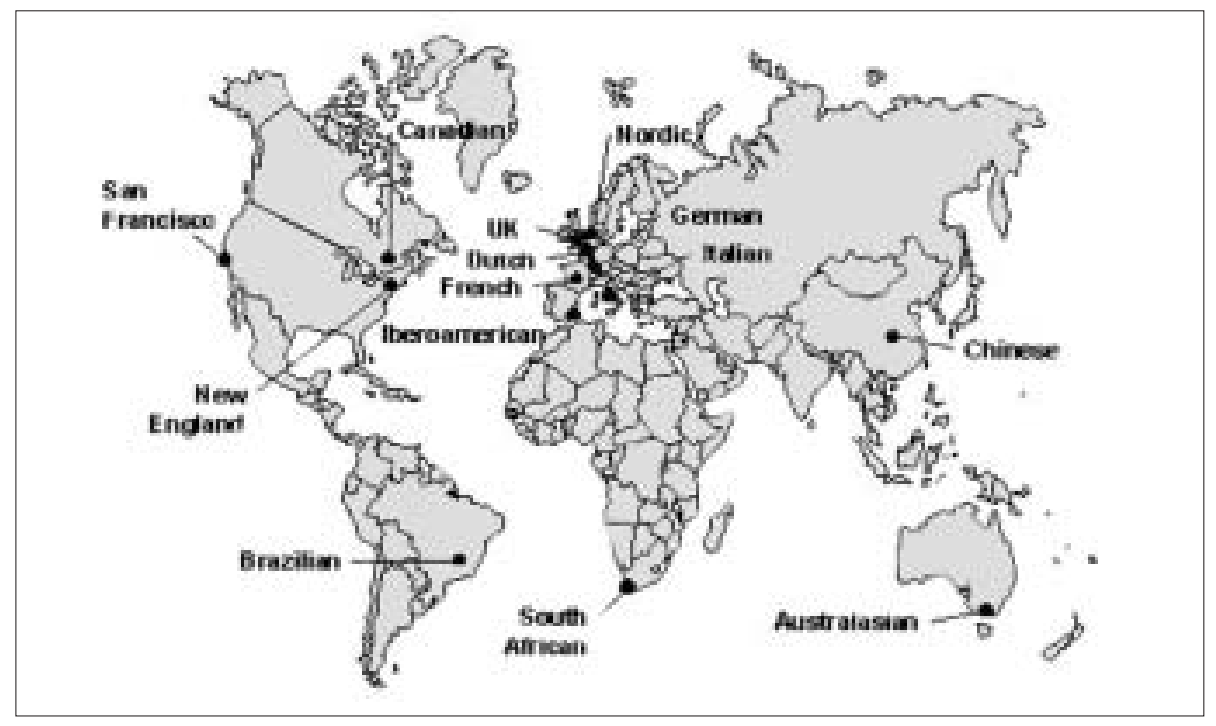

Figura 1. Centros Cochrane.

nes Cochrane. En la actualidad también podemos acceder a Cochrane Library plus en español, que coordinada por Infoglobal Suport es, por el momento, la única base de datos Cochrane en un idioma distinto al inglés. Con estas revisiones sistemáticas se pretende minimizar los elementos de arbitrariedad propios de las revisiones tradicionales, detallando minuciosamente el proceso de revisión aplicado para que otra persona pueda realizarlo y comprobar la veracidad de las conclusiones. Sin embargo, se trata de una sistemática de trabajo laboriosa y en expansión por lo que se explica que muchos de los temas que pueden interesar a una especialidad como la Cirugía Oral y Maxilofacial no estén, por el momento contemplados. Entre los diferentes grupos colaboradores de revisión Cochrane figura el Cochrane Oral Health Group, donde se recogen diversas revisiones sobre implantología, líquen plano, síndrome de boca ardiente, etc. A pesar de todo, si pretendiéramos dar respuesta al interrogante clínico que nuestro enfermo con su tumor parotídeo nos había planteado, observaremos que mediante la red Cochrane, no podríamos obtener una solución. Asimismo, se han desarrollando por parte de las diferentes sociedades y especialidades médicas, guías y protocolos de práctica clínica, algunos de ellos basados en pruebas, y de fácil acceso por Internet. Por otra parte, la introducción en la medicina de los denominados sistemas expertos, programas informáticos dotados de conocimiento y comportamiento próximos a los de un experto humano, ${ }^{17}$ puede potenciar y facilitar la metodología de la $\mathrm{MBE}$, siempre que la incorporación de conocimientos a la base de datos que maneje el programa (LogicTree ${ }^{\circledR}$, Resolver ${ }^{\circledR}$ ) siga los protocolos de revisión sistemática postulados por la MBE.

\section{Evaluación crítica de la evidencia}

Tras la selección y lectura de un trabajo con el que se pretende dar respuesta al interrogante clínico planteado, deberemos plantearnos el grado de validez de los resultados obtenidos. ${ }^{18}$ Por un lado, deberemos tener en cuenta la validez interna del trabajo, es decir hasta qué punto los resultados del estudio reflejan la realidad de la población estudiada; de otra parte deberemos tener en consideración su validez externa, es decir, la posibilidad de extrapolar los resultados del estudio a poblaciones diferentes a la que se realizó. ${ }^{19}$ Para el ejercicio de este análisis crítico el profesional de la salud debe disponer, al menos de una manera básica, de ciertos conocimientos sobre estadística y diseño de investigación. La información proporcionada por los centros de MBE, permiten al profesional disponer de un conocimiento revisado y confrontado que elimina sesgos y garantiza una mayor fiabilidad, con menores conocimientos sobre disciplinas ajenas a su ejercicio clínico. ${ }^{20,21}$ El mejor conocimiento para el diagnóstico provendrá de los indicadores probabilísticos derivados de los estudios de pruebas diagnósticas para obtener su sensibilidad, especificidad, valores predictivos, cocientes de probabilidades, etc. Para efectuar un pronóstico, el mejor conocimiento se obtendrá de apropiados estudios de seguimiento (estudios de cohortes). Para las decisiones terapéuticas, el conocimiento de mayor calidad proviene de los ensayos clínicos con distribución aleatoria, que aportan las probabilidades de obtener un resultado de interés en un grupo de pacientes tratados con determinado procedimiento, intervención o sustancia, en relación a un tratamiento alternativo o a un placebo (estudio clínico randomizado). El nivel de evidencia sobre la cuestión planteada variará en 


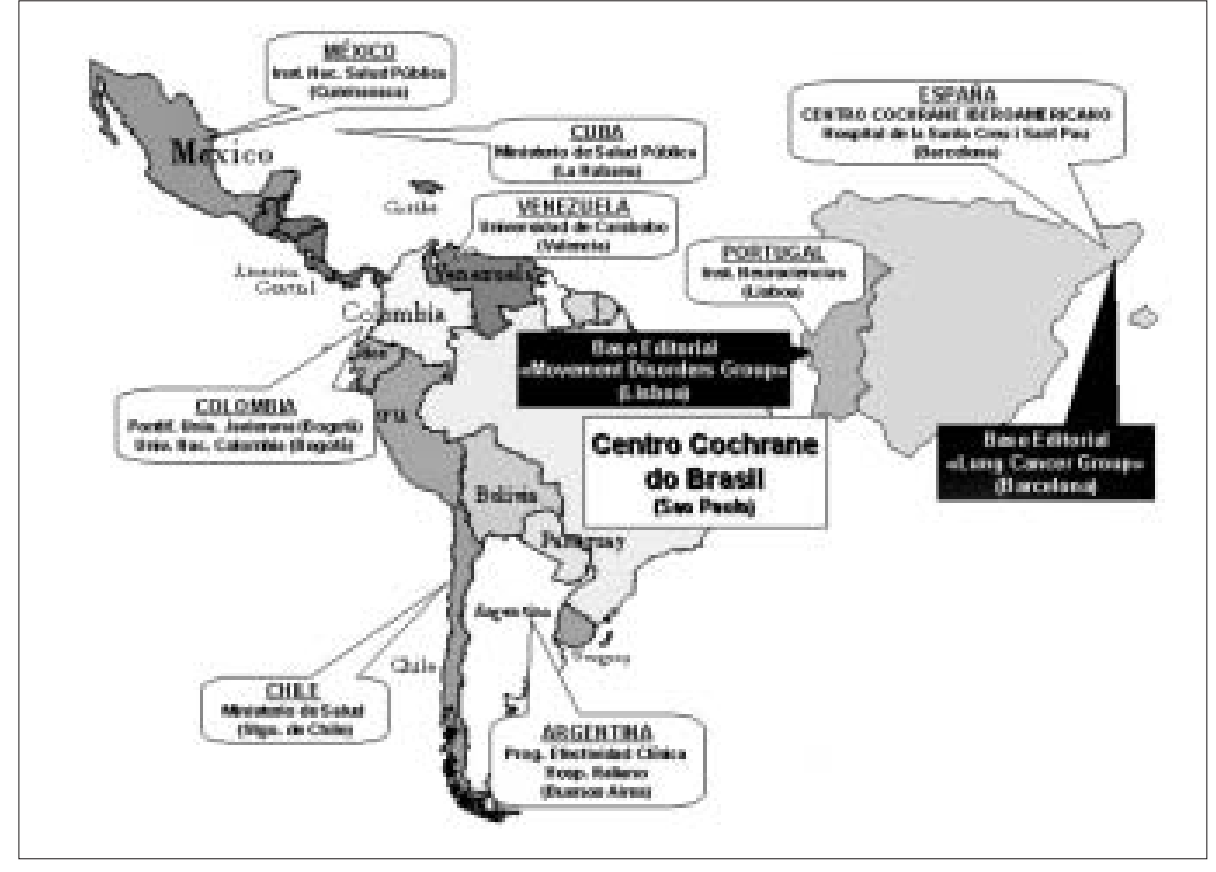

Figura 2. La colaboración Cochrane Iberoamericana. función del diseño y metodología de los estudios que la establecen (Tabla 3).

\section{Aplicación de las conclusiones de esta evaluación a la práctica}

La información obtenida tras la consulta de revisiones sistemáticas, debe ser individualizada para el contexto personal del enfermo que generó el interrogante. Por ejemplo, si la mejor prueba de diagnóstico de imagen para un tumor de parótida es la resonancia magnética, pero por nuestra historia clínica sabemos que el enfermo padece claustrofobia, la solicitud por nuestra parte de este estudio complementario retardará un diagnóstico de manera imprudente. Vuelve a ponerse de manifiesto con este ejemplo, que la MBE también depende de forma importante de la experiencia y habilidad clínica del profesional que la desarrolla.

\section{Discusión}

La MBE es una nueva corriente para la práctica médica justificada por tres grandes realidades. La primera de ellas se fundamenta en la imposibilidad de conocer todo lo que hoy en día podríamos conocer, situación que no es nueva, ya que Leibnitz la describió magistralmente hace casi 300 años: «ya nadie puede saber lo que hay que saber». La segunda tiene que ver con la profusión de la información biomédica existente en la actualidad, recogida en más de 25.000 revistas y más de 1.600 artículos de medicina al día, la
Tabla 3. Niveles de evidencia

1. Evidencia bien fundada, al menos en una revisión sistemática de varios estudios randomizados controlados correctamente diseñados.

2. Evidencia bien fundada, al menos en un estudio randomizado controlado correctamente diseñado y del tamaño adecuado.

3. Evidencia de estudios no randomizados, estudios de un único grupo antes y después de una intervención, estudios de cohortes, de series cronológicas y de casos y controles.

4. Evidencia de estudios no experimentales correctamente diseñados con más de un centro o grupo de investigadores.

5. Opiniones de autoridades respetadas o de comité de expertos, basadas en evidencias clínicas o estudios descriptivos.

mayoría sin aportaciones significativas para el conocimiento. ${ }^{22}$ La tercera tiene que ver con la expansión del acceso a la información médica a través de Internet con más de 500 millones de usuarios en todo el mundo. ${ }^{23}$ Estas tres situaciones justificaron y/o potenciaron el desarrollo del paradigma propio de la MBE que tiene como centro de referencia la validación de la información médica mediante estudios sistemáticos de los contenidos bibliográficos. ${ }^{24}$ Desde el punto de vista filosófico, esta nueva tendencia constituye una buena aportación al ejercicio práctico de la medicina, pero puede que realmente su objetivo princeps no sea tan novedoso como propugnan sus defensores. Grahamme-Smith, ${ }^{25}$ se ha referido al «disentimiento socrático»sobre la Medicina Basada en la Evidencia en un artículo muy comentado. En supuesto diálogo con Sócrates, el entusiasta de la medicina basada en la evi- 
dencia, Entusiasticus (meta-analiticus), señala que hoy se desarrolla una nueva y muy efectiva forma de práctica médica, denominada Medicina Basada en la Evidencia. Responde Sócrates: «Creía que todos los médicos eran formados en la tradición científica, uno de cuyos baluartes es el examen de la evidencia sobre la cual fundamentan su práctica. ¿En qué forma, entonces, difiere esta nueva medicina basada en la evidencia de la medicina tradicional??. Responde Entusiasticus: Bueno, Sócrates, un problema consiste en que la mayoría de los médicos trabajan en una perspectiva demasiado estrecha, confiando en su propia experiencia y en la de unos pocos colegas con quienes intercambian opiniones. Ello los conduce, en ocasiones, a conclusiones erróneas». A la pregunta de Sócrates sobre si esto es una expresión del conflicto entre la ciencia y el arte de la medicina, Entusiasticus responde que quizás es así, y que él mismo se halla tan obsesionado con esta metodología, que ya no tiene tiempo para versus pacientes.

En nuestra opinión, ha sido la revolución en la tecnología de acceso a la información la que ha permitido llevar hacia delante la implantación de la metodología de la MBE. Pongamos un ejemplo, si hace menos de 14 años nos propusiéramos en nuestro país una revisión bibliográfica sobre el síndrome de boca ardiente, deberíamos por empezar consultando manualmente los listados bibliográficos (Index Medicus, Index Dental, Cumulated Index, Current Contents, Core Journals, etc.) sobre el tema existentes en las bibliotecas de hospitales y/o universidades. A continuación, deberíamos proceder a la obtención de todos los trabajos y después a la selección de aquellos que pudieran ser incluidos en el estudio. Esta labor nos podría ocupar fácilmente varios meses. En la actualidad todo este proceso es susceptible de realización en siete días, y la primera parte del mismo en segundos. Por tanto la MBE surgió y se desarrolló en el momento histórico en el que la tecnología permitió su origen y desarrollo. Por otra parte, no puede obviarse que MBE continúa manteniendo la necesaria existencia de la una medicina teórica basada en la experimentación. Por ejemplo, si Branemark no hubiera demostrado experimentalmente la oseointegración de los implantes de titanio, todas las aportaciones ulteriores que la MBE desarrolló a este respecto, no existińan. Por otra parte, desde esta perspectiva filosófica no podemos obviar que la clásica afirmación de la medicina Aristotélica sobre el saber particular (saber práctico: no hay certeza, sólo probabilidad) se mantiene de algún modo en la MBE. De lo contrario, carecenía de sentido la periódica actualización de resúmenes que las diferentes organizaciones de MBE llevan a cabo. Por ejemplo, la realización del vaciamiento cervical radical según la técnica de Crile fue sustituido, para determinados casos, por el vaciamiento funcional de Suárez, y éste a su vez, y también para determinados casos, por los vaciamientos supraomohiodeos; pero las posibilidades de cambio por «mejores evidencias» siguen abiertas. Por tanto, las mejores evidencias»sobre un tema concreto del año 2003, pueden ser diferentes e incluso antagónicas a las que se obtengan en el año 2006. A largo plazo estaríamos hablando de una nueva comiente dentro de la MBE, la MBE de la nueva evidencia o contra-la evidencia.

Se ha postulado por parte de algunos detractores, que la MBE genera un mayor gasto sanitario. Es dificil mantener esta afirmación hoy en día. La tecnología necesaria para su uso está prácticamente generalizada en los centros sanitarios (si bien con deficiencias y estrecheces, en muchos casos), y no cabe duda de que una mejor selección en los estudios complementarios y en la aplicación de los tratamientos, redundará en un mejor resultado para el paciente y para el sistema sanitario. Sólo es un problema de formación y no de economía. Sin embargo, no podemos dejar de reconocer que, hoy por hoy, la MBE es una medicina para «ricos», en la medida en la que sólo un $10 \%$ de la población mundial dispone de tecnología y medios económicos (o sus países) para una atención sanitaria digna. En este sentido, la MBE no es más que el reflejo en el mundo sanitario del movimiento de globalización de nuestro tiempo, y por analogía a éste conlleva, al menos inicialmente, un ahondamiento en las diferencias socio-sanitarias. En nuestro mundo el ejercicio médico según las directrices postuladas por la MBE, no es una opción si no una obligación, como ya no es una opción, solicitar la realización de una sialografía para el diagnóstico de un tumor parotídeo. Esta realidad ha sido contemplada por muchos profesionales, que rápidamente se han apuntado a la moda de defender una MBE sin un ejercicio personal de la misma. Este tipo de posturas no favorecen la promoción ni los objetivos propuestos por la MBE. Tampoco favorecen su desarrollo las restricciones del lenguaje derivadas de la exclusiva elaboración de preguntas que puedan ser verificadas (sustracción metodológica). ${ }^{26,27}$ Por otra parte, es sabido que los seres humanos tienen tendencia a creer en los fenómenos sensoriales sobre cualquier otro tipo de información (fenomenología), lo que implica la inexistencia de una conexión automática entre verificación (validación) y voluntad de aplicación. Finalmente, la denominada disonancia cognitiva, tendencia natural a aferrarse a una creencia a pesar de que la evidencia muestre su falsedad, es otro de los obstáculos que la metodología de la MBE debe superar. Desde el punto de vista técnico y educativo, aunque el sistema de enseñanza basado en problemas ha sido incorporado por diversas Universidades extranjeras, en nuestro país la reforma de los planes de estudio de la Licenciatura de Medicina que se fueron sucediendo a lo largo de la década de los noventa, pretendieron una mayor interrelación entre las ciencias 
básicas y clínicas, pero no han logrado ni aproximarse a un tipo de enseñanza afín con el paradigma de la MBE. Es por ello, que las fuentes de aprendizaje para esta nueva metodología del ejercicio médico procedan de cursos de post-grado o de la inquietud personal autodidacta del profesional. Tampoco las autoridades sanitarias han promovido, en nuestro país, políticas de divulgación e implementación de la MBE, quizá por la incertidumbre de rentabilidad económica que les inspira. Contrasta esta postura con la mantenida por la Administración frente a la denominada Cirugía Mayor Ambulatoria, y es un reflejo más de que en política sanitaria priman más los efectos de gestión económica que los de praxis médica. A este respecto, Anderson, ${ }^{28}$ denunciaba en Science la gran paradoja que debe afrontar cualquier reforma del sistema sanitario: «os gestores de la sanidad que deseen alentar a los médicos a aplicar las prácticas clínicas con un mejor balance entre coste y beneficio deben afrontar un gran problema: los mismos médicos, por no mencionar a los técnicos ministeriales, no saben frecuentemente cuáles son, entre tantas, las prácticas más eficaces». Para seguir la metodología propuesta por la MBE, el profesional debe sustituir, en aras de una mayor pertinencia y validez, unas fuentes de información fáciles de obtener, por otras que implican búsquedas bibliográficas y valoración crítica. Podría parecer que este proceso es largo y costoso, pero Sharon y Straus, ${ }^{29}$ demuestran que, en menos de 10 minutos, se puede obtener información consistente sobre interrogantes clínicos concretos (empleo de la espirolactona en insuficiencia cardíaca congestiva p. ej.), accediendo a la información de tres fuentes bibliográficas solventes (New England Journal Medicine, ACP Journal Club Online y Clinical Evidence). El principal obstáculo que, a nuestro juicio, limita actualmente un rápido ejercicio de la MBE, es que no siempre, y especialmente en Cirugía Oral y Maxilofacial, la literatura médica tiene las repuestas para las decisiones que se deben tomar en la práctica o éstas no están disponibles tras revisiones sistemáticas. Para ser francos, en nuestra Especialidad el cuerpo doctrinal que la MBE ha testado es mínimo, por lo que el camino por recomer es enorme. Lo mismo sucede con otras especialidades quirúrgicas. El porcentaje de decisiones fundamentadas en estudios clínicos randomizados en la denominada Cirugía Basada en la Evidencia (CBE), no supera el 20\%, mientras que en la medicina interna no invasora este porcentaje alcanza el $50 \% .30$

Los estudios clínicos randomizados tienen serias limitaciones en el contexto de los tratatamientos quirúrgicos, estimándose que sólo el 39\% de éstos pueden ser evaluados mediante este tipo de estudios.31,32 Por otra parte, la necesidad de acceso mediante abono de suscripción a las bases de datos MBE, así como el hecho de que muchas revistas biomédicas continúen primando su divulgación convencional frente a la online, tampoco facilita la sistemática de trabajo propuesto por la MBE. Los cambios que nos esperan en este apartado serán imparables, a pesar de los intereses económicos que los frenan.

\section{Conclusiones}

En definitiva, el paciente que acude a nuestra consulta busca en nosotros el calor humano inherente a la profesión médica, pero también una respuesta científica a sus problemas. La MBE es en la actualidad un instrumento apropiado para hacer frente a esta última cuestión, y su implantación es ya una obligación para el profesional de la salud, que terminará viendo su ejercicio como algo natural, consustancial a su profesión. Las observaciones personales, tan frecuentemente expresadas «en mi experiencia»irán perdiendo peso por la información sistematizada obtenida de la comunidad científica.

\section{Agradecimientos}

Al Dr. Martín-Granizo por su amable invitación para la elaboración del presente trabajo.

\section{Bibliografía}

1. Guyat G. Preface. En: Guyatt G, Rennie D (eds.) User's Guide to the Medical Literature. Essentials of Evidenced Medicine Clinical Practice. AMA Press, EE.UU. 2002.

2. Sackett DL, Rosenberg W, Muir JA, Haynes RB, Richardson WS. Evidence based medicine: what it is and what it isn't. BMJ 1996;312:71-2.

3. Ortiz Z, García Dieguez M, Laffaire M. Medicina Basada en la Evidencia. Boletín de Academia de Medicina de Buenos Aires. bvs.insp.mx/componen/mbevid/bibcoch/ doc/MBE.

4. Gracia D. Medicina basada en la evidencia: aspectos éticos. Bioética 2000;8:1. www.cfm.org.br/revista/bio1v8/ simpo3.htm

5. Marshall T. Scientific knowledge: a new clinical epistemology? J Eval Clin Pract 1997;3:133-5.

6. Cohen L. McMaster's pioneer in evidence-based medicine now spreading his message in England. Can Med Assoc J 1996;154:388-90.

7. Bonfill X, Gabriel R, Cabello J. La medicina basada en la evidencia. Rev Esp Cardiol 1997;50:819-25.

8. Bonfill X. La Colaboración Cochrane. Jano 1997;52:63-5.

9. Chalmers I, Dickersin K, Chalmers TC. Getting to grips with Archie Cochrane's agenda. BMJ 1992;305:786-9.

10. Introduction to Evidence Based Medicine. EBM Tool KitIntroduction to Informatics. www.med.ualberta.ca/ebm/ ebmintro.htm. 
11. Bravo Toledo R, Campos Asensio C. Medicina basada en pruebas. Jano 1997;1218:71-2.

12. Rosenberg W, Donald A. Evidence based medicine: an approach to clinical problem-solving. BMJ 1995;310:11226.

13. Evidence-Based Medicine Working Group. EvidenceBased Medicine. A new approach to teaching the practice of medicine. JAMA 1992;268:2420-5.

14. Medicina Basada en la Evidencia. www.infodoctor.org/ rafabravo/mbe.htm

15. Introduction to Evidence-Based Medicine. www.hsl.unc. $\mathrm{edu} / \mathrm{lm} / \mathrm{ebm} /$

16. La red Cochrane Iberoamericana (Iberoamerican Cochrane Network). www.cochrane.es/castellano/CC-Resumen

17. Baladrón Romero J. Sistemas Expertos en Cirugía del Esqueleto Facial. Tesis Doctoral. Universidad de Oviedo 1999.

18. Greenhalgh T. Cómo interpretar un artículo médico. Fundamentos de la medicina basada en la evidencia. Medical Trenes, SL. Barcelona, 2000.

19. Dans AI, Dans LF. The need and means for evidencebased medicine in developing countries. Evidence Based Medicine 2000;5:100-1.

20. Knottnerus J, Dinant G. Medicine based evidence, a prerequisite for evidence based medicine BJM 1997;315: 13.

21. Oxman AD, Sackett DL, Guyatt GH. Users' guides to the medical literature. I. How to get started. JAMA 1993;270: 2093-95.
22. Jefferson TO, Alderson P, Davidoff F, Pager Editorial peerreview for improving the quality of reports of biomedical studies. The Cochrane Library 2003;1. www.cochranelibrary.com.

23. Martín-Granizo R. Revistas biomédicas virtuales: cel final de la era del papel?. Rev Esp Cirug Oral y Maxilofac 2002; 24:221.

24. Guerra Romero L. La medicina basada en la evidencia: un intento de acercar la ciencia al arte de la práctica clínica. Med Clin (Barc) 1996;107:377-82.

25. Grahamme-Smith D. Evidence based medicine: Socratic dissent. BMJ 310:1126,1995.

26. Ortega M, Cayuela A. Medicina basada en la evidencia: una crítica filosófica sobre su aplicación en atención primaria. Rev Esp Salud Pública 2002;76:115-20.

27. Locutura J, Lorenzo JF. El lenguaje médico español. La realidad y el deseo. Med Clin 2003;120:424-5.

28. Anderson C. Measuring what works in health care. Science 1994;263:1.080-82.

29. Sharon E, Straus MD. Evidence-based medicine in practice. Evidence-Based Medicine 2002;7:68-9.

30. Kenny SE, Shankar KR, Rintala R, Lamond GL, Lloyd DA. Evidence-based surgery: interventions in a regional paediatric surgical unit. Arch Dis Child 1997;76:50-3.

31. García-Caballero M, Neugebauer E, Sauerland S, Lefering R. Ventajas e inconvenientes de la cirugía basada en la evidencia. Cir Esp 2000;67:486-96.

32. Solomon MJ, McLeod RS. Should we be performing more randomized controlled trials evaluating surgical operations?. Surgery 1995;118:459-67. 